\title{
VISCOELASTICITY OF DYNAMICALLY FIXED BIOPROSTHETIC VALVES. II. EFFECT OF GLUTARALDEHYDE CONCENTRATION
}

Anthony C. Duncan, $\mathrm{PhD}^{\mathrm{a}}$

Derek Boughner, $\mathrm{PhD}, \mathrm{MD}^{\mathrm{a}}$

Ivan Vesely, $\mathbf{P h D}^{\mathrm{b}}$
Objectives: We have previously shown the benefits of dynamic fixation over conventional static fixation of bioprosthetic valves. In an attempt to increase the durability of bioprosthetic heart valves, we explored the benefit of low-concentration glutaraldehyde dynamic fixation. Methods: Pig aortic valves obtained fresh from the abattoir and excised with the entire root were dynamically fixed in glutaraldehyde phosphate buffer solutions varying in concentration from $0.05 \%$ to $2.5 \%$. Denaturation temperatures were measured and mechanical testing was performed at low $(3 \mathrm{~mm} / \mathrm{sec})$ to high physiologic rates $(30 \mathrm{~mm} / \mathrm{sec})$ at $37^{\circ} \mathrm{C}$ in isotonic modified Hanks solution. Results: When fixed dynamically in $0.05 \%$ glutaraldehyde solution for 24 hours, the tissue reached a degree of cross-linking (denaturation temperature $=82.8^{\circ} \pm 0.6^{\circ} \mathrm{C}$ ) significantly higher than that obtained for $0.05 \%$ static fixation (denaturation temperature $\left.=79.3^{\circ} \pm 0.9^{\circ} \mathrm{C}\right)(p<0.05)$ but similar to that for conventional static fixation in $0.5 \%$ glutaraldehyde solution (denaturation temperature $=83.5^{\circ} \pm 0.3^{\circ} \mathrm{C}$ ). After fixation in low-concentration glutaraldehyde $(0.05 \%)$, final relaxation slopes and moduli in the circumferential direction were significantly higher than those for the statically fixed tissue but similar to those for the fresh tissue. However, both dynamic and static fixation had the effect of increasing tissue extensibility to similar extents in both directions, irrespective of glutaraldehyde concentration. Conclusion: Dynamic glutaraldehyde fixation of a porcine aortic valve at lower concentrations resulted in a better degree of cross-linking and a material with biomechanical properties that more closely mimic those of natural heart valve tissue. ( $J$ Thorac Cardiovasc Surg 1997;113:302-10)
Cor more then two decades glutaraldehyde has $F$ been used in the treatment of porcine aortic valve tissue for bioprosthetic substitutes of diseased heart valves. ${ }^{1-4}$ Although glutaraldehyde stabilization has

From J. P. Robarts Research Institute, University of Western Ontario, London, Canada, ${ }^{a}$ and The Cleveland Clinic Foundation, Cleveland, Ohio. ${ }^{b}$

Supported in part by a grant from the Medical Research Council of Canada (MRC). Dr Anthony C. Duncan was supported by a postdoctoral fellowship from the MRC, Dr. D. Boughner is a Research Associate at the Heart and Stroke Foundation of Ontario, Canada, and Dr. I. Vesely is a Research Scholar of the Foundation.

Received for publication April 2, 1996; revisions requested May 22, 1996; revisions received Sept. 27, 1996; accepted for publication Oct. 8, 1996.

Address for reprints: Anthony C. Duncan, J. P. Robarts Research Institute, 100 Perth Dr., PO Box 5015, London, Ontario, N6A $5 \mathrm{~K} 8$ Canada.

Copyright (C) 1997 by Mosby-Year Book, Inc.

$0022-5223 / 97 \$ 5.00+0 \quad \mathbf{1 2 / 1 / 7 8 5 0 6}$ led to valves with satisfactory hemodynamics, low thrombogenicity, ${ }^{2}$ and reduced antigenicity, ${ }^{1,3}$ ironically problems associated with the use of glutaraldehyde have limited their long-term performance. These problems include leaflet calcification, possible cytotoxicity caused by unreacted glutaraldehyde reagent, and alteration of the natural biomechanical properties of the valve. Although it was initially believed that tensile stresses induced during valve closure were responsible for cuspal tearing, studies have shown that rupture is related more to flexural fatigue. ${ }^{5,6}$

Shear deformations normally contribute to a reduction in the compressive and tensile stresses developed under flexion and likely represent the natural stress-reducing mechanism of the valve. Internal shearing is a mechanism by which the aortic valve can reduce internal tensile and compressive stresses generated by bending during the cyclic opening and closure of the valve. 
We hypothesize that glutaraldehyde fixation disables this natural stress-reducing mechanism of the valve as a result of shearing limitations associated with collagen interfiber cross-linking. We proposed dynamic pulsatile fixation as an alternative solution to preserve the natural, stress-relieving shear mechanism of the fresh valve tissue by allowing collagen fiber or bundles (or both) to be free to move and rearrange in a sterically favorable configuration. In previous studies, we have shown that dynamically fixed valve cusps sheared five times more than the statically fixed tissue. ${ }^{7}$ To date, there have been to our knowledge only two articles reporting dynamic fixation of valves as an alternative treatment. 8,9

$\mathrm{We}^{10}$ have previously demonstrated the ability of dynamic fixation to preserve important biomechanical properties of porcine bioprosthetic aortic valve tissue. In contrast with conventional static fixation, which considerably alters the viscoelastic properties of the material, stress-relaxation slopes and tissue modulus remained unaffected after dynamic fixation in specific conditions (i.e., low pressure and low pulsation rates). Consistent with previous data for static fixation, dynamic fixation at low hydrostatic pressures (i.e., below $4 \mathrm{~mm} \mathrm{Hg}$ ) is expected to preserve the natural crimp of collagen and is thereby more conducive to mimic the natural biomechanical properties of fresh (untreated) tissue. ${ }^{11}$

The purpose of this study is to further investigate dynamic fixation as a possible alternative to effectively fix bioprosthetic porcine aortic valve tissue using lower concentrations of glutaraldehyde. This would limit adverse effects associated with conventional static fixation (i.e., calcification, toxicity). The dynamic fixation method was also proposed as an effective method to offset limitations associated with conventional static fixation. These include limited diffusion of the reagent into the valve matrix, surface polymerization of glutaraldehyde, and poor accessibility of reactive collagen sites to the glutaraldehyde reagent.

\section{Materials and methods}

Dynamic fixation. Pig aortic valves were obtained fresh from the abattoir, excised with the entire root and right coronary muscular shelf, and placed immediately in Hanks solution before being processed. The valves were tested or processed within 24 hours of retrieval. The fixation tank was filled with a phosphate-buffered solution $(0.2 \mathrm{~mol} / \mathrm{L}, \mathrm{pH} 7.4)$, and a valve was connected to the apparatus and preconditioned for 15 minutes at a pulsation rate of $1.2 \mathrm{~Hz}$, as described previously. ${ }^{10} \mathrm{~A}$ total of 69 porcine aortic valves were cut out with their aortic root and divided into three groups: 30 were fixed dynamically with a low transmural cyclic pressure (varying from 0 to 4 $\mathrm{mm} \mathrm{Hg}$ ) at the same pulsation rate used during the preconditioning procedure, 30 were fixed statically, and nine were tested fresh. The dynamically fixed valves were divided into six groups of five each: three groups fixed in $0.05 \%, 0.5 \%$, and $2.5 \%$ glutaraldehyde-phosphate solutions for two different durations ( 3 and 24 hours). While one valve was fixed dynamically, another was fixed statically in the same tank, at a low hydrostatic transvalvular pressure gradient (i.e., below $4 \mathrm{~mm} \mathrm{Hg}$ ) and used as a control. Each valve yielded one cusp for circumferential and one for radial testing (the third cusp was stored or used for denaturation temperature determination). Fixation was performed at room temperature. Although wellshaped leaflets were produced and the valve remained closed throughout most of the fixation period, the valve did open intermittently over a short period of time in the early stages of the dynamic fixation. The pressure gradient for the hydrostatically fixed valves was sufficient to maintain them closed during the entire fixation period. A correlation of closed valve fixation with in vivo performance has not been fully established, and open valve fixation has been suggested to be more favorable. ${ }^{12}$

Glutaraldehyde was purchased from BDH Chemicals, Toronto, Ontario, Canada, $50 \% \mathrm{w} / \mathrm{w}$. The valves were stored in a $0.01 \% \mathrm{w} / \mathrm{v}$ gentamicin-sterilant modified Hanks solution $(0.2 \mathrm{~mol} / \mathrm{L}, \mathrm{pH} 7.4)$ at $4^{\circ} \mathrm{C}$ for further use. Although storage over periods of 12 to 16 months appeared to have little effect on the tensile properties of the valve tissue (e.g., modulus, ultimate tensile strength, and stress relaxation rate), ${ }^{13}$ care was taken to minimize the delay between fixation and tensile testing (i.e., no more than 2 to 3 weeks).

Denaturation temperature determination

Principle. Glutaraldehyde fixation results in resistance to thermal or biodegradation caused by the stabilization of structural collagen by internal cross-linking. Collagen stabilization has currently been measured by in vivo preservation, in vitro resistance to enzymatic degradation, or thermal stability. Thermal stability measures the temperature at which the tertiary helical hydrogen-bonded structure of collagen collapses to a random coil..$^{10}$ The temperature at which this occurs is the denaturation temperature and is characteristic of the degree of crosslinking and stabilization of collagen. ${ }^{10}$

The denaturation temperature can be determined by three methods: (1) differential scanning calorimetry, which is used more generally for the study of transitions on a wide range of materials, (2) shrinkage temperature tests, and (3) hydrothermal isometric tests. The latter two are unique to the study of biologic collagenous materials. In the shrinkage temperature test the material is held under a constant load (isotonic) in a heated water bath with the temperature rising at a constant rate (about $2^{\circ} \mathrm{C} / \mathrm{min}$ ) while length is monitored over time. Shrinkage begins when denaturation temperature is reached. In the hydrothermal isometric test method, the material is held at a constant length (isometric) and allowed to relax. The force is monitored over time and decreases monotonically until denaturation temperature is reached. At this point the load curve reverses owing to a pull of the denatured, 
rubbery collagen. At the onset of the denaturation process, the more thermally stable chemical bonds remain, to yield a cross-linked gel of denatured collagen. As the temperature of collagen rises above denaturation temperature, collagen denaturation proceeds and the crosslinked collagen network continues to shrink, thus generating the characteristic pull in the direction opposite that of initial loading, and load increases monotonically. The temperature at the inflexion point of the curve of force versus temperature (or time) is the denaturation temperature.

Denaturation temperatures were measured with the use of an apparatus described previously. ${ }^{10}$ Each sample was extended to a given load (about 200g) and allowed to relax at constant length (isometric) in a temperature-controlled water bath. Temperature was raised at a rate of $2^{\circ} \mathrm{C} / \mathrm{min}$. Weight and temperature were monitored as a function of time and stored on an interfaced personal computer with the use of a Quick Basic acquisition program (Microsoft Quick Basic, version 4.5).

A total of four samples per group were tested for the denaturation temperatures $(n=4)$. Denaturation temperature data for the $2.5 \%$ glutaraldehyde-treated samples are not shown here because we were interested mainly in determining the ability of this new fixation technique to cross-link collagen at lower concentrations of glutaraldehyde.

In addition, previous studies have shown that when cusp tissue is exposed to such high concentrations $(2.5 \%)$ of glutaraldehyde, the maximum degree of cross-linking is reached long before the 3.25 to 24 hours considered in our study (i.e., within minutes) ${ }^{14}$; therefore no differences between statically fixed and dynamically fixed tissue would be anticipated. Because commercial valves are never treated in such high concentrations of glutaraldehyde (i.e., $2.5 \%$ ) but rather at much lower concentrations (e.g., $0.5 \%$ ), the degree of cross-linking at high concentrations was examined.

Mechanical testing. Two cusps were excised from each aortic root (five per group of fixed tissue and nine for the fresh tissue group). Each cusp was cut into rectangles of $2 \times 1 \mathrm{~cm}$ and $2 \times 0.5 \mathrm{~cm}$ for mechanical testing in the circumferential and radial directions, respectively. ${ }^{10,15,16}$

Preconditioning. Preconditioning was performed on each strip before testing, by cyclic loading from $250 \mathrm{~g}$ (circumferential strips) or $150 \mathrm{~g}$ and above (radial strips), at the same strain rate used for the mechanical testing experiment. Preconditioning was continued until a reproducible, identical curve was obtained over several cycles. This was generally the case after roughly 30 cycles. Load versus elongation was monitored and plotted simultaneously on the computer screen for immediate verification.

Tensile testing. Mechanical testing was performed with a hydraulically powered MTS machine (MTS, Minneapolis, Minn.), coupled with a data acquisition system. Samples were tested at $37^{\circ} \mathrm{C}$ in an isotonic modified Hanks buffer $(0.2 \mathrm{~mol} / \mathrm{L}, \mathrm{pH} 7.4)$ using a special apparatus designed and built in our laboratory. ${ }^{17}$

Both stress-strain and stress-relaxation tests were performed at extension rates of 3 and $30 \mathrm{~mm} / \mathrm{sec}$. The 30 $\mathrm{mm} / \mathrm{sec}$ extension rate was characteristic of physiologic in vivo strain rates (i.e., $15,000 \% \min ^{-1}$ ). ${ }^{13,}{ }^{15-18}$ The 3 $\mathrm{mm} / \mathrm{sec}$ extension rate was based on previous tensile testing experiments ${ }^{10,13,15-18}$ and is of the same order of magnitude as the fastest strain rates achieved in the past for tensile testing. ${ }^{13,17}$ After conditioning, stress-strain curves were obtained by straining the sample to roughly the preconditioning load and acquiring load versus extension. The stress relaxation was performed immediately after the stress-strain test (at the same extension rate), while the sample was still mounted and preconditioned. This involved stretching the tissue strip to a similar load and maintaining the sample stretched at constant length while monitoring load decay versus time (over 100 seconds).

Strips of 5 and $10 \mathrm{~mm}$ width were cut from the valve leaflets for testing in the circumferential and radial directions, respectively, by means of a surgical blade and a template with variable widths. Each aortic valve provided two cusps for both directions; the third cusp was used for denaturation temperature determination. Thickness was measured with a custom-built and Mitutuyo (model IDC112CE; Mitutuyo Corporation, Japan) thickness gauge described previously. ${ }^{15}$

During testing, the end of each strip was placed between the serrated jaws of a top and bottom grip. ${ }^{10}$ The bottom grip was immobilized to the MTS desktop whereas the top grip could move freely in the vertical direction. The gauge length of the tissue was considered to be the length at which the tissue was straightened and the load began to deviate from zero. The mean gauge length was about $5.0 \pm 1.0 \mathrm{~mm}$ and $8.0 \pm 1.0 \mathrm{ram}$ (mean \pm standard deviation) for the radial and circumferential strips, respectively.

Data analysis. The engineering stress was measured as the load normalized for the area and defined as:

$$
\sigma=\frac{\mathrm{Lxg}}{\mathrm{txW}}
$$

where $\sigma$ is stress in kilopascals, $\mathrm{L}$ is the load in kilograms recorded by the load cell, $\mathrm{g}$ is the acceleration of gravity constant equal to $9.81 \mathrm{~N} / \mathrm{kg}$, and $\mathrm{t}$ and $\mathrm{W}$ are the initial thickness and width of the tissue specimen in millimeters, respectively.

Engineering strain was defined as:

$$
\epsilon=\frac{1-\lg }{\lg }
$$

where $\epsilon$ is the strain in percent, $l$ is the length, and $\lg$ is the gauge length of the sample at zero load.

Stress relaxation was plotted as a percent of stress remaining versus time on a decimal logarithmic scale. The percent stress remaining was calculated as follows:

$$
\text { Percent stress remaining }=\frac{\sigma(\mathrm{t})}{\sigma(0)} \times 100
$$

where $\sigma(t)$ and $\sigma(0)$ were the engineering stresses measured at time $t$ and initially (at $t=0$ ), respectively. The relaxation curves of percent stress remaining versus $\log$ of time were fit by two linear regression lines. One linear regression was used to fit the data in the range from 


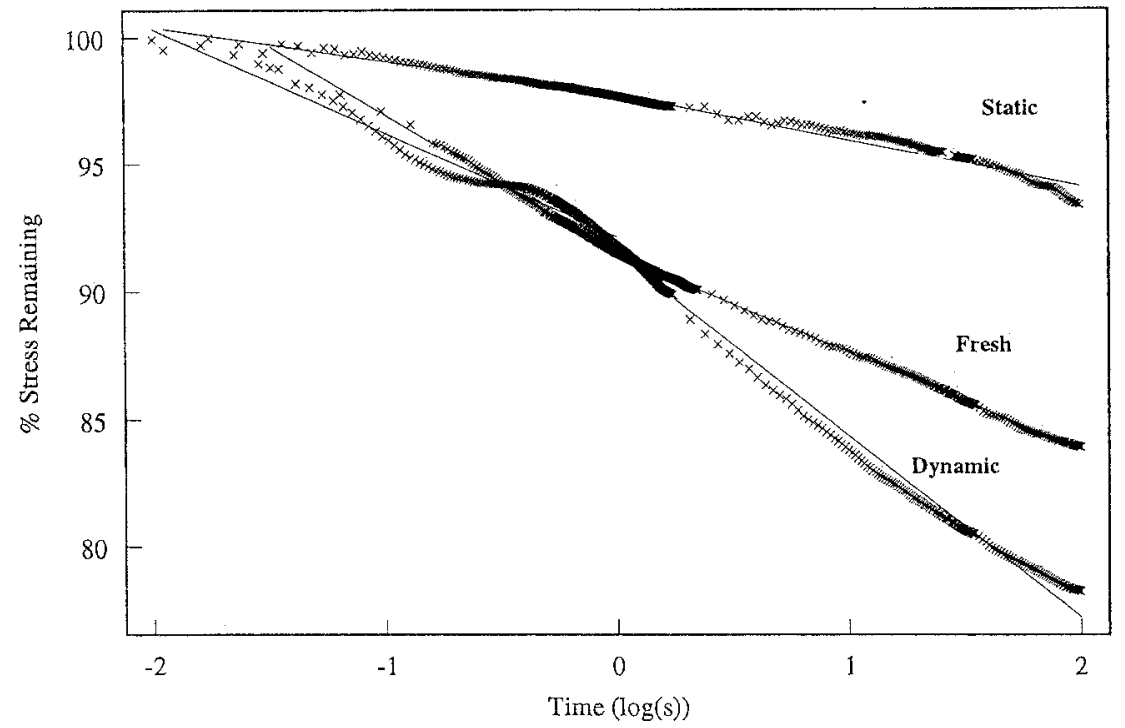

Fig. 1. Stress-relaxation curves showing a two-piece linear regression fit for each curve representative of preconditioned circumferential fresh, dynamically fixed, and statically fixed porcine aortic valve tissue (at a $30 \mathrm{~mm} / \mathrm{sec}$ extension rate).

0.01 to 1 second; the slope of the line provided the initial relaxation slope. Another linear regression was used to fit the data in the range from 1 to 100 second; the slope of the second fit provided the final relaxation slope.

Relaxation curves representative of the three tissues with the two-piece linear fit are shown in Fig. 1. Although the initial portion of the curve was not always perfectly fit by a straight line in both directions, it was believed that the initial phase of the relaxation curve was different from the final one. All the data (except for two curves) provided a good fit $\left(R^{2}>0.98\right)$ for the initial phase of the relaxation curve. Stress-strain curves representative of the different samples tested in the circumferential direction are shown in Fig. 2. Modulus was obtained by measuring the slope of the tangent to the stress-strain curve at a given stress value (400 $\mathrm{kPa}$ and $100 \mathrm{kPa}$ for the circumferential and radial directions, respectively). Extensibility was determined as the strain value at which the corresponding tangent line intersected with the $x$-axis (i.e., strain axis).

\section{Results}

\section{Denaturation temperatures}

Effect of fixation time. The results of the denaturation temperatures for fresh, statically fixed, and dynamically fixed valves are shown in Table I. When fixed in $0.5 \%$ glutaraldehyde, no further increase in the denaturation temperature was observed beyond 3 hours 15 minutes of fixation, for both dynamically and statically fixed samples, suggesting that the cross-linking reaction with glutaraldehyde was complete within that time. This was consistent with previous data for the statically fixed valve tissue, since Woodroof ${ }^{14}$ showed that a plateau for the denaturation temperature was reached (at roughly $83^{\circ} \mathrm{C}$ ) beyond 3 hours 15 minutes of fixation when fixed in a $0.5 \%$ glutaraldehyde solution. At that duration, denaturation temperature for the dynamically treated valve was significantly higher $\left(89.5^{\circ} \pm\right.$ $0.3^{\circ} \mathrm{C}$ ) than that for the statically treated valve $\left(83.5^{\circ} \pm 0.3^{\circ} \mathrm{C}\right)$ or the fresh (untreated) tissue $\left(65.6^{\circ} \pm 0.8^{\circ} \mathrm{C}\right)$, suggesting a higher degree of collagen cross-linking for the dynamically fixed tissue.

At low glutaraldehyde concentrations (i.e., $0.05 \%$ ), longer durations (i.e., 24 hours) were required to reach complete fixation (i.e., denaturation temperature equal to roughly $83^{\circ} \mathrm{C}$ ). After 24 hours, dynamic fixation in $0.05 \%$ glutaraldehyde, however, reached a degree of cross-linking (denaturation temperature $=82.8^{\circ} \pm 0.6^{\circ} \mathrm{C}$ ) equivalent to that for the conventional statically fixed tissue in $0.5 \%$ glutaraldehyde (denaturation temperature $=83.5^{\circ} \pm$ $0.3^{\circ} \mathrm{C}$ ). In addition, when fixed long enough (i.e., 24 hours), dynamic fixation in a $0.05 \%$ glutaraldehyde solution significantly increased the degree of crosslinking over that obtained by the standard static fixation procedure $(p<0.05)$ (Table I).

\section{Tensile testing}

Stress relaxation. The results for the final stressrelaxation slopes are presented in Table II, as a function of fixation duration for the fresh, statically 


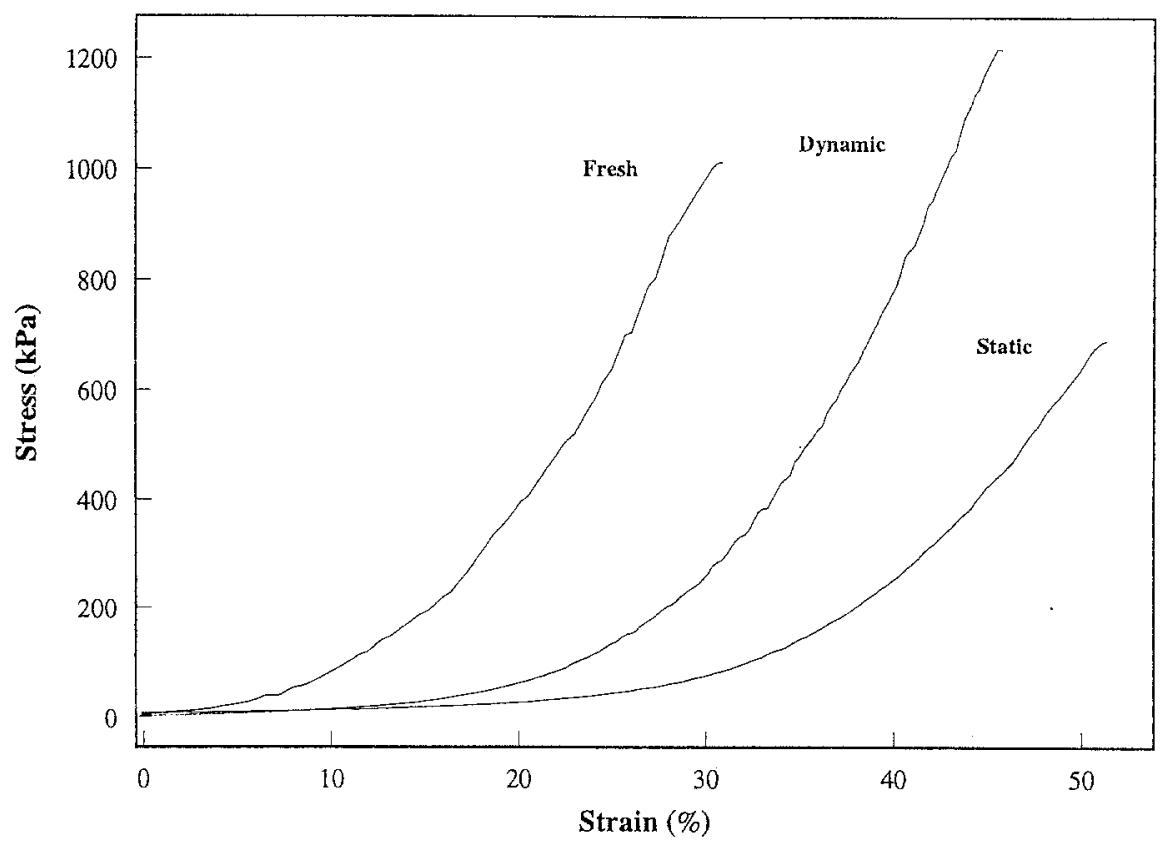

Fig. 2. Set of stress-strain curves representative of preconditioned circumferential fresh, dynamically fixed, and statically fixed porcine aortic valve tissue (at a $3 \mathrm{~mm} / \mathrm{sec}$ extension rate).

Table I. Hydrothermal isometric temperatures $\left({ }^{\circ} \mathrm{C}\right)$ for fresh (untreated), statically fixed, and dynamically fixed valves $(n=4)$

\begin{tabular}{lll}
\hline & \multicolumn{2}{c}{ Glutaraldehyde concentration } \\
\cline { 2 - 3 } Time $(h r)$ & $0.05 \%$ & $0.5 \%$ \\
\hline 3.25 & & \\
Static & $75.2(0.4)$ & $83.5(0.3)$ \\
$\quad$ Dynamic & $77.9(1.1)$ & $89.5(0.3)^{*}$ \\
24 & & \\
$\quad$ Static & $79.3(0.9)$ & $83.8(0.9)$ \\
$\quad$ Dynamic & $82.8(0.6)^{*}$ & $89.7(0.1)^{*}$ \\
Fresh, 65.6 $(0.08)$ & & \\
\hline
\end{tabular}

Values are mean (standard deviation).

*Significant difference between statically fixed and dynamically fixed tissue $(p<0.05)$

fixed, and dynamically fixed valves. In the circumferential direction after 3 hours 15 minutes of fixation, significant differences in the final relaxation slopes were observed between dynamically and statically fixed tissue at both low $(0.05 \%)$ and normal $(0.5 \%)$ glutaraldehyde concentrations (Table II). No differences were observed between statically and dynamically fixed tissue when fixed in normal $0.5 \%$ glutaraldehyde solutions for 24 hours or when fixed at high concentrations (i.e., $2.5 \%$ ). After 24 hours, differences between dynamic and static fixation were significant when valves were fixed in low concentrations of glutaraldehyde (i.e., $0.05 \%$ ). In the radial direction, no differences between dynamically and statically fixed tissue were observed for the final relaxation slopes, except for fixation in a normal $0.5 \%$ glutaraldehyde solution ( 3 hours 15 minutes).

Initial relaxation slopes are shown in Table III. In the circumferential direction, significant differences were found between statically and dynamically fixed tissue at low concentrations $(0.05 \%)$ after 24 hours' fixation. Initial relaxation slopes for dynamically fixed tissue were significantly higher and approximated those for the (untreated) fresh tissue. In the radial direction, initial relaxation slopes were similar for either statically or dynamically fixed tissue.

Stress-strain response. The stress-strain parameters for tensile testing in the circumferential and radial direction are presented in Table IV. In the circumferential direction, significant differences were seen in the modulus values between statically and dynamically fixed valves for low $(0.05 \%)$ and intermediate $(0.5 \%)$ concentrations when fixed completely (i.e, 24 hours and 3 hours 15 minutes and beyond, respectively). No such differences were seen at a higher concentration $(2.5 \%)$. In the radial direction, we did not observe differences in the elastic tensile modulus of dynamically and statically fixed tissue, except in the case of tissue fixed in a 
Table II. Final stress-relaxation slopes for fresh, statically fixed, and dynamically fixed tissue at $1.2 \mathrm{~Hz}$ in $0.05 \%, 0.5 \%$, and $2.5 \%$ glutaraldehyde phosphate buffer in the circumferential and radial directions $1 \%$ stress remaining versus $\log [\mathrm{sec}]$ )

\begin{tabular}{|c|c|c|c|c|c|c|}
\hline & \multicolumn{2}{|c|}{$0.05 \%$} & \multicolumn{2}{|c|}{$0.5 \%$} & \multicolumn{2}{|c|}{$2.5 \%$} \\
\hline & $30 \mathrm{~mm} / \mathrm{sec}$ & $3 \mathrm{~mm} / \mathrm{sec}$ & $30 \mathrm{~mm} / \mathrm{sec}$ & $3 \mathrm{~mm} / \mathrm{sec}$ & $30 \mathrm{~mm} / \mathrm{sec}$ & $3 \mathrm{~mm} / \mathrm{sec}$ \\
\hline \multicolumn{7}{|c|}{ Circumferential direction } \\
\hline \multicolumn{7}{|c|}{$3 \mathrm{hr} 15 \mathrm{~min}$} \\
\hline Static & $-3.4(0.3)^{*}$ & $-3.9(0.6)^{*}$ & $-3.9(1.7)^{*}$ & $-5.4(2.5)$ & $-3.5(0.7)$ & $-4.5(1.1)$ \\
\hline Dynamic & $-6.4(0.9)$ & $-7.1(1.4)$ & $-7.4(0.7)$ & $-5.4(2.5)$ & $-4.2(0.9)$ & $-5.2(1.3)$ \\
\hline \multicolumn{7}{|l|}{$24 \mathrm{hr}$} \\
\hline Static & $-2.3(0.5) \dagger$ & $-3.2(0.8)^{*}$ & $-5.3(0.8)$ & $-3.1(0.7)$ & & \\
\hline Dynamic & $-9.7(1.9)$ & $-5.8(3.3)$ & $-4.4(2.1)$ & $-2.9(1.4)$ & & \\
\hline Fresh & $-5.8(1.3)$ & $-6.5(1.9)$ & & & & \\
\hline \multicolumn{7}{|c|}{ Radial direction } \\
\hline \multicolumn{7}{|c|}{$3 \mathrm{hr} 15 \mathrm{~min}$} \\
\hline Static & $-4.8(0.3)$ & $-5.4(0.7)$ & $-4.3(0.7)^{*}$ & $-5.8(2.4)$ & $-3.9(0.2)$ & $-6.1(1.5)$ \\
\hline Dynamic & $-5.3(1.8)$ & $-4.8(0.4)$ & $-7.3(1.2)$ & $-5.1(0.8)$ & $-4.5(1.4)$ & $-5.3(0.7)$ \\
\hline \multicolumn{7}{|l|}{$24 \mathrm{hr}$} \\
\hline Static & $-3.4(0.2)$ & $-5.7(1.1)$ & $-6.3(0.7)$ & $-4.3(0.6)$ & & \\
\hline Dynamic & $-4.3(0.8)$ & $-5.0(0.2)$ & $-5.3(1.0)$ & $-6.1(0.8)$ & & \\
\hline Fresh $^{*}$ & $-5.3(1.8)$ & $-7.6(0.9)$ & & & & \\
\hline
\end{tabular}

Mean (standard deviation). $n=5$ for the fixed samples; $n=9$ for fresh tissue. Differences are significant at $* p<0.05$ and $\$ p<0.02$.

Table III. Initial stress-relaxation slopes for fresh, statically fixed, and dynamically fixed tissue at $1.2 \mathrm{~Hz}$ in $0.05 \%, 0.5 \%$, and $2.5 \%$ glutaraldehyde phosphate buffer in the circumferential and radial directions (\% stress remaining versus $\log [\mathrm{sec}])$

\begin{tabular}{|c|c|c|c|c|c|c|}
\hline & \multicolumn{2}{|c|}{$0.05 \%$} & \multicolumn{2}{|c|}{$0.5 \%$} & \multicolumn{2}{|c|}{$2.5 \%$} \\
\hline & $30 \mathrm{~mm} / \mathrm{sec}$ & $3 \mathrm{~mm} / \mathrm{sec}$ & $30 \mathrm{~mm} / \mathrm{sec}$ & $3 \mathrm{~mm} / \mathrm{sec}$ & $30 \mathrm{~mm} / \mathrm{sec}$ & $3 \mathrm{~mm} / \mathrm{sec}$ \\
\hline \multicolumn{7}{|c|}{ Circumferential direction } \\
\hline \multicolumn{7}{|c|}{3 hr 15 min } \\
\hline Static & $-6.3(0.9)$ & $-3.8(0.5)$ & $-7.8(1.2)$ & $-4.5(1.6)$ & $-6.1(1.4)$ & $-5.1(1.2)$ \\
\hline Dynamic & $-7.6(0.6)$ & $-2.9(0.8)$ & $-5.6(1.1)$ & $-4.4(2.0)$ & $-6.6(1.1)$ & $-5.6(0.7)$ \\
\hline \multicolumn{7}{|l|}{$24 \mathrm{hr}$} \\
\hline Static & $-5.6(1.4)$ & $-5.1(1.8)$ & $-9.8(1.8)$ & $-3.7(3.0)$ & & \\
\hline Dynamic & $-12.4(2.2) \div$ & $-4.5(1.9)$ & $-9.0(1.2)$ & $-5.7(0.8)$ & & \\
\hline Fresh & $-8.1(1.4)$ & $-6.0(2.0)$ & & & & \\
\hline \multicolumn{7}{|c|}{ Radial direction } \\
\hline \multicolumn{7}{|c|}{$3 \mathrm{hr} 15 \mathrm{~min}$} \\
\hline Static & $-11.8(1.0)$ & $-7.3(1.8)$ & $-6.9(0.6)$ & $-9.6(1.5)$ & $-6.9(1.2)$ & $-5.0(0.2)$ \\
\hline Dynanic & $-10.8(1.6)$ & $-4.8(0.4)$ & $-10.0(1.0)$ & $-3.2(0.7)$ & $-7.1(0.7)$ & $-4.5(0.3)$ \\
\hline \multicolumn{7}{|l|}{$24 \mathrm{hr}$} \\
\hline Static & $-9.2(1.6)$ & $-6.1(0.9)$ & $-9.0(1.0)$ & $-6.1(0.6)$ & & \\
\hline Dynamic & $-9.2(1.2)$ & $-6.9(2.0)$ & $-10.5(1.6)$ & $-6.3(0.8)$ & & \\
\hline Fresh* & $-7.0(2.6)$ & $-5.9(1.5)$ & & & & \\
\hline
\end{tabular}

Mean (standard deviation). $n=5$ for the fixed samples; $n=9$ for fresh tissue. Differences are significant at $* p<0.05$ and $\neq p<0.001$.

$0.5 \%$ glutaraldehyde solution for 3 hours 15 minutes. In both directions the modulus for the glutaraldehyde-fixed valves was smaller, on average, than that of fresh tissue.

The extensibility values are presented in Table V. Both dynamic and static fixation had the effect of increasing tissue extensibility in both directions at all concentrations studied. However, no significant dif- ferences were detected between statically and dynamically fixed valves at all concentrations studied. With the exception of the statically fixed tissue in the circumferential direction, extensibility of tissue fixed at high glutaraldehyde concentrations (i.e., 2.5\%) was significantly higher in both directions than that of tissue fixed at the low glutaraldehyde concentrations (i.e., $0.05 \%)(p<0.01)$. 
Table IV. Tensile modulus for fresh, statically fixed, and dynamically fixed tissue in $0.05 \%, 0.5 \%$, and $2.5 \%$ glutaraldehyde phosphate buffer in the cirumferential and radial direction (MPa)

\begin{tabular}{|c|c|c|c|c|c|c|}
\hline & \multicolumn{2}{|c|}{$0.05 \%$} & \multicolumn{2}{|c|}{$0.5 \%$} & \multicolumn{2}{|c|}{$2.5 \%$} \\
\hline & $30 \mathrm{~mm} / \mathrm{sec}$ & $3 \mathrm{~mm} / \mathrm{sec}$ & $30 \mathrm{~mm} / \mathrm{sec}$ & $3 \mathrm{~mm} / \mathrm{sec}$ & $30 \mathrm{~mm} / \mathrm{sec}$ & $3 \mathrm{~mm} / \mathrm{sec}$ \\
\hline \multicolumn{7}{|c|}{ Circumferential direction } \\
\hline \multicolumn{7}{|c|}{$3 \mathrm{hr} 15 \mathrm{~min}$} \\
\hline Static & $5.2(3.8)$ & $5.0(2.8)$ & $4.3(1.7)^{*}$ & $5.0(1.8)^{*}$ & $1.1(0.2)$ & $1.4(0.1)$ \\
\hline Dynamic & $4.5(2.0)$ & $4.1(2.2)$ & $8.7(3.8)$ & $8.2(3.6)$ & $1.4(1.0)$ & $1.6(1.3)$ \\
\hline \multicolumn{7}{|l|}{$24 \mathrm{hr}$} \\
\hline Static & $0.7(0.1) \dagger$ & $1.1(0.1) \dagger$ & $0.5(0.1)^{*}$ & $0.4(0.1)^{*}$ & & \\
\hline Dynamic & $3.7(1.4)$ & $4.5(0.3)$ & $2.0(0.4)$ & $1.8(0.9)$ & & \\
\hline Fresh & $5.9(2.5)$ & $5.7(2.4)$ & & & & \\
\hline \multicolumn{7}{|c|}{ Radial direction } \\
\hline \multicolumn{7}{|c|}{$3 \mathrm{hr} 15 \mathrm{~min}$} \\
\hline Static & $1.0(0.5)$ & $2.0(0.7)$ & $0.2(0.0)^{*}$ & $0.2(0.1)^{*}$ & $0.3(0.1)$ & $0.4(0.1)$ \\
\hline Dynamic & $2.2(1.3)$ & $2.5(1.6)$ & $1.4(0.1)$ & $1.1(0.1)$ & $0.3(0.1)$ & $0.3(0.1)$ \\
\hline \multicolumn{7}{|l|}{$24 \mathrm{hr}$} \\
\hline Static & $1.4(0.5)$ & $1.3(0.7)$ & $0.9(0.1)$ & $0.8(0.2)$ & & \\
\hline Dynamic & $1.7(1.1)$ & $3.0(1.3)$ & $0.4(0.1)$ & $0.5(0.1)$ & & \\
\hline Fresh & $0.5(0.1)$ & $0.6(0.1)$ & & & & \\
\hline
\end{tabular}

Mean (standard deviation). $n=5$ for the fixed samples; $n=9$ for fresh tissue. Differences are significant at " $p<0.05$ and $\dagger p<0.02$.

Table V. Extensibilities for fresh, statically fixed, and dynamically fixed tissue in $0.05 \%, 0.5 \%$, and $2.5 \%$ glutaraldehyde phosphate buffer in the circumferential and radial directions

\begin{tabular}{|c|c|c|c|c|c|c|}
\hline & \multicolumn{2}{|c|}{$0.05 \%$} & \multicolumn{2}{|c|}{$0.5 \%$} & \multicolumn{2}{|c|}{$2.5 \%$} \\
\hline & $30 \mathrm{~mm} / \mathrm{sec}$ & $3 \mathrm{~mm} / \mathrm{sec}$ & $30 \mathrm{~mm} / \mathrm{sec}$ & $3 \mathrm{~mm} / \mathrm{sec}$ & $30 \mathrm{~mm} / \mathrm{sec}$ & $3 \mathrm{~mm} / \mathrm{sec}$ \\
\hline \multicolumn{7}{|c|}{ Circumferential direction } \\
\hline \multicolumn{7}{|l|}{$3 \mathrm{hr} 15 \mathrm{~min}$} \\
\hline Static & $30.0(10.0)$ & $30.5(13.8)$ & $37.5(7.8)$ & $41.0(9.3)$ & $51.2(7.3)$ & $58.6(10.0)$ \\
\hline Dynamic & $41.5(4.5)$ & $42.5(21.5)$ & $23.1(9.0)$ & $26.9(8.9)$ & $60.6(17.9)$ & $67.2(29.3)$ \\
\hline \multicolumn{7}{|l|}{$24 \mathrm{hr}$} \\
\hline Static & $30.7(10.0)$ & $50.1(12.0)$ & $40.0(10.0)$ & $41.0(9.1)$ & & \\
\hline Dynamic & $32.5(10.0)$ & $54.7(30.0)$ & $46.0(3.7)$ & $53.4(3.0)$ & & \\
\hline Fresh & $13.2(3.6)$ & $11.2(3.2)$ & & & & \\
\hline \multicolumn{7}{|c|}{ Radial direction } \\
\hline \multicolumn{7}{|l|}{3 br $15 \mathrm{~min}$} \\
\hline Static & $47.7(14.7)$ & $59.0(4.4)$ & $58.0(9.6)$ & $68.0(8.8)$ & $98.4(27.9)$ & $130.0(6.3)$ \\
\hline Dynamic & $52.8(3.2)$ & $75.9(17.3)$ & $95.7(35.8)$ & $79.3(6.0)$ & $105.4(13.8)$ & $129.3(17.8)$ \\
\hline \multicolumn{7}{|l|}{$24 \mathrm{hr}$} \\
\hline Static & $65.5(17.3)$ & $63.43(9.0)$ & $54.2(6.5)$ & $53.7(6.9)$ & & \\
\hline Dynamic & $56.5(18.3)$ & $51.0(21.0)$ & $32.0(17.4)$ & $54.3(5.5)$ & & \\
\hline Fresh & $39.5(11.3)$ & $38.2(9.9)$ & & & & \\
\hline
\end{tabular}

Data are expressed as mean (standard deviation). $n=5$ for the fixed samples; $n=9$ for fresh tissue.

On average, extensibility in the radial direction was higher than that in the circumferential direction for both fresh and fixed tissue, consistent with previous data. ${ }^{10,13,16,17}$

\section{Discussion}

We have previously shown that dynamic fixation could be used as an alternative treatment to produce a bioprosthetic valve with mechanical advantages over the conventional statically fixed valves. This was attributed to the ability of the process to offset reduction in the natural internal shearing ability of porcine aortic valve tissue while optimizing the cross-linking reaction with collagen. In this article we have examined the hypothesis that with dynamic fixation lower concentrations of glutaraldehyde could be used to fix bioprosthetic heart valve tissue with similar or superior cross-linking efficiency. Dynamic fixation could thus reduce the risks associated with the use of high levels of glutaraldehyde. 
We chose a low concentration of $0.05 \%$, because fixation was deemed to be complete and stable after 24 hours, as indicated by the denaturation temperatures (see Table I). Consistent with previous data for heart valve tissue, ${ }^{14}$ cross-linking was shown to be complete for concentrations all the way down to $0.1 \%$ when the valves were fixed overnight.

On the basis of the denaturation temperature measurements, fixation in $0.5 \%$ glutaraldehyde was complete within 3 hours 15 minutes; longer durations were required to fix porcine aortic heart valves in $0.05 \%$ glutaraldehyde for both dynamic and static fixation. After 24 hours, dynamic fixation in both low and normal concentrations of glutaraldehyde significantly increased denaturation temperature compared with that for static fixation, suggesting a higher degree of internal cross-linking for the dynamically fixed tissue. This is consistent with the fact that dynamic fixation may result in a better penetration of the glutaraldehyde reagent and facilitate the availability of more potential reactive sites on the collagen molecules or fibers (or both) that would normally have remained unexposed under static fixation conditions, as suggested previously. ${ }^{10}$

In the circumferential direction (i.e., that of greatest collagen fiber alignment), valves dynamically fixed in $0.05 \%$ and $0.5 \%$ glutaraldehyde showed greater final stress-relaxation rates than the statically fixed tissue. Although these differences remained at low glutaraldehyde concentrations (i.e., $0.05 \%$ ), they appeared to dissipate after longer fixation times at an intermediate concentration (i.e., 24 hours at $0.5 \%$ ) or at a high concentration (i.e., $2.5 \%$ ). The reason for this is unclear but may involve a greater penetration of unreacted glutaraldehyde reagent at longer durations (i.e., 24 hours) that may, in turn, dissipate any differences in biomechanics observed previously for lower durations.

At both low $(0.05 \%)$ and intermediate concentrations $(0.5 \%)$, dynamic fixation significantly increased the circumferential tensile modulus of the tissue to values that more closely mimic the natural (untreated) heart valve tissue. No differences in modulus were observed between dynamically and statically fixed tissue in the radial direction.

For fixation at 3 hours 15 minutes and beyond, extensibility of tissue fixed in both low and high concentrations was higher than that of fresh tissue. However, extensibilities in both directions were similar for both statically and dynamically fixed tissue.

\section{Conclusion}

Dynamic glutaraldehyde fixation of a porcine aortic valve at low concentrations provided a tissue with properties similar to that of the fresh aortic valve tissue (i.e., stress-relaxation slopes and elastic modulus closer to that of the natural valve tissue). This could present an advantage over the conventional static fixation technique, in that similar and qualitatively better cross-linking can be achieved with lower concentrations of glutaraldehyde. The technique could lead to a valve with biomechanics that more closely mimic those of fresh (untreated) valve tissue, with a greater degree of cross-linking and possibly a greater preservation of the important natural internal shearing ability of the valve tissue. We anticipate that this technique will allow for fixation of bioprosthetic heart valve tissue at much lower concentrations than those used in the conventional static fixation technique. This technique may also limit the risks associated with conventional static glutaraldehyde fixation (i.e., calcification, toxicity) and thus lead to a valve with potentially improved long-term biomechanics and durability. The long-term performance and biostability of such a porcine aortic valve tissue ultimately will be assessed by pulse duplicator tests and in vivo animal studies.

\section{REFERENCES}

1. Cohn LH, Collins JJ Jr. The glutaraldehyde stabilized porcine aortic valve. In: Ionescu MI, editor. Tissue heart valves. Toronto: Butterworths, 1979:173-200.

2. Roberts WC. Substitute cardiac valves. Adv Cardiol 1978;22: 252-70.

3. Cohn LH, Gilbert HM, Pratter F, Collins JJ Jr. Five to eight year follow-up of patients undergoing porcine heart-valve replacement. N Engl J Med 1981;304:258-62.

4. Angell WW, Angell JD, Kosek JC. Clinical and experimental comparisons establishing the glutaraldehyde treated xenografts as the standard for tissue heart valve replacement. In: Ionescu MI, editor. Tissue heart valves. Toronto: Butterworths, 1979:89-126

5. Vesely I, Boughner D, Song T, Tissue buckling as a mechanism of bioprosthetic valve failure. Ann Thorac Surg 1988; 346:302-8.

6. Fung FC. Bioviscoelastic solids. In: Fung FC, editor. Biomechanics: mechanical properties of living tissues, 2nd ed. New York: Springer Verlag, 1993:242-51.

7. Song T, Vesely I, Boughner D. Effects of dynamic fixation on shear behaviour of porcine xenograft valves. Biomaterials 1990;11:191-6.

8. Mavrilas D, Missirlis Y. An approach to the optimization of preparation of bioprosthetic heart valves. J Biomech 1991; 24:331-9.

9. Purinya B, Kasyanov V, Volkolakov J, Latsis R, Tetere G. 
Biomechanical and structural properties of explanted bioprosthetic valve leaflets, J Biomech 1994;27:1-11.

10. Duncan AC, Boughner D, Vesely I. Dynamic fixation of a porcine aortic valve xenograft. I. Effect of fixation conditions on the final viscoelastic properties. Biomaterials 1996;17: 1849-56.

11. Broom ND, Thompson FJ. Influence of fixation conditions on the performance of glutaraldehyde-treated porcine aortic valves: towards a more scientific basis. Thorax 1979;34:166-76.

12. Imamura $\mathrm{E}$, Ishihara S, Ohteki H, Aomi S, Koyanagi H. Open-position fixation of bioprostheses for more physiological performance. J Thorac Cardiovasc Surg 1984;88:114-21.

13. Lee JM, Courtman DW, Boughner DR. The glutaraldehydestabilized porcine aortic valve xenograft. II. Effect of fixation with or without pressure on the tensile viscoelastic properties of the leaflet material. J Biomed Mater Res 1984;18:79-98.

14. Woodroof EA. The chemistry and biology of aldehyde treated tissue heart valve xenografts. In: Ionescu MI, editor. Tissue heart valves. Toronto: Butterworths, 1979;347-62.

15. Leeson-Dietrich J, Boughner D, Vesely I. Porcine pulmonary and aortic valves: a comparison of their tensile visco elastic properties at physiological strain rates. J Heart Valve Dis 1995;4:88-94.

16. Leeson-Dietrich JM, Vesely I, Boughner DR. Bioprosthetic valve tissue viscoelasticity: implications on accelerated pulse duplicator testing. Ann Thorac Surg 1995;60(2 Suppl):S37982; discussion $\$ 383$.

17. Lee JM, Courtman DW, Boughner DR. The glutaraldehydestabilized porcine aortic valve xenograft. I. Tensile viscoelastic properties of the fresh leaflet material. J Biomed Mater Res 1984;18:61-77.

18. David H, Boughner DR, Vesely I, Gerosa G. The pulmonary valve: Is it mechanically suitable for use as an aortic valve replacement? Trans ASAIO 1994;40:206-12.

\section{Bound volumes available to subscribers}

Bound volumes of The Journal of Thoracic and Cardiovascular Surgery are available to subscribers (only) for the 1997 issues from the Publisher, at a cost of $\$ 110.50$ for domestic, $\$ 139.64$ for Canadian, and $\$ 130.50$ for international subscribers for Vol. 113 (January-June) and Vol. 114 (July-December). Shipping charges are included. Each bound volume contains a subject and author index and all advertising is removed. Copies are shipped within 60 days after publication of the last issue of the volume. The binding is durable buckram with the Journal name, volume number, and year stamped in gold on the spine. Payment must accompany all orders. Contact Mosby-Year Book, Inc., Subscription Services, 11830 Westline Industrial Drive, St. Louis, Missouri 63146-3318, USA; phone $800-453-4351$ or $314-453-4351$.

Subscriptions must be in force to qualify. Bound volumes are not available in place of a regular Journal subscription. 